\title{
Families of irreptiles
}

Christian Richter

Christian Richter absolvierte seine mathematische Ausbildung bis zur Habilitation im Jahr 2001 an der Friedrich-Schiller-Universität Jena. Derzeit stellt ihm die Deutsche Forschungsgemeinschaft ein Heisenberg-Stipendium für geometrische und approximationstheoretische Studien in Jena und Paris zur Verfügung.

\section{Introduction}

The search for tilings of the plane by congruent images of some given polygon $A$ leads in a natural way to the concept of a reptile. $A$ is called a reptile if it can be dissected into finitely many pairwise congruent pieces which are similar images of $A$. We speak of a dissection if the covering pieces can only have boundary points in common.

Many known examples of reptiles are polyominoes or polyiamonds. A polygon is called a polyomino (polyiamond) if it has a connected interior and possesses an edge-to-edge dissection into finitely many congruent squares (equilateral triangles).

A family of reptiles that are polyominoes can be obtained as follows (see [2], [3, p. 97], [6, p. 54], and the first illustration in Fig. 1). Fix an integer $k \geq 1$ and dissect a square $S$ into $(2 k)^{2}$ congruent smaller squares $S_{1}, \ldots, S_{(2 k)^{2}}$. Let $\delta_{c}$ denote the rotation about the centre $c$ of $S$ by an angle of $\frac{\pi}{2}$. Now choose a simple polygonal arc $\Gamma$ contained in the union of the boundaries of the pieces $S_{i}$ that connects $c$ with a point on the boundary of $S$ such that $\Gamma \cap \delta_{c}(\Gamma)=\{c\}$. Then $\Gamma, \delta_{c}(\Gamma)$, and a quarter of the boundary of $S$ bound a reptile $A \subseteq S$ (shaded in Fig. 1). Indeed, since $A$ splits into $k^{2}$ congruent squares $S_{i}$ and since $S$ as well as any other square admits a dissection into four congruent similar copies of $A, A$ can be

Ein Polygon $P$ wird selbstähnlich genannt, wenn es als Vereinigung von $n \geq 2$ innendisjunkten ähnlichen Kopien seiner selbst darstellbar ist. Durch Iteration der Zerlegungsprozedur erlaubt jedes solche $P$ Partitionen in endlich viele beliebig kleine ähnliche Bilder von $P$. Umgekehrt gestattet $P$ Pflasterungen der Ebene durch Einbettung in immer grössere ähnliche Kopien. In der vorliegenden Arbeit beschreibt der Autor Konstruktionsprinzipien für gewisse Klassen selbstähnlicher Polygone. Die Prinzipien sind durch unterhaltungsmathematische Studien zu Polyominos und Polyiamonds motiviert, führen aber deutlich über diesen Rahmen hinaus. 

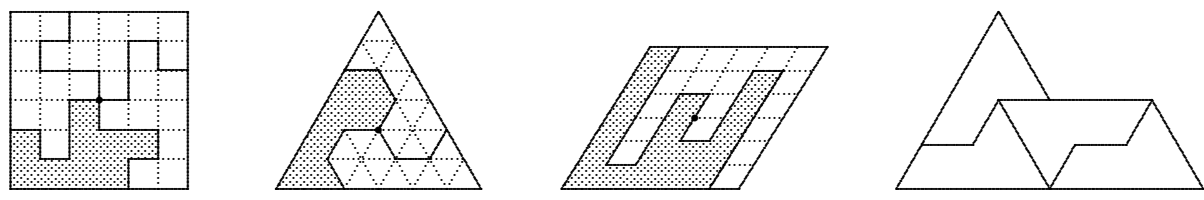

Fig. 1 Examples of reptiles

dissected into $4 k^{2}=(2 k)^{2}$ pairwise congruent images of $A$ under suitable similarities. A similar procedure starting with an equilateral triangle gives a family of reptiles that are polyiamonds (see [2], [6, p. 175], and the second part of Fig. 1).

The third family illustrated in Fig. 1 contains reptiles obtained from an arbitrary parallelogram $P$. One splits $P$ into $(2 k)^{2}$ congruent smaller similar copies $P_{1}, \ldots, P_{(2 k)^{2}}$ and fixes a simple polygonal arc $\Gamma$ connecting two points of the boundary of $P$ and symmetric with respect to the centre of $P$ that is contained in the union of the boundaries of the pieces $P_{i}$. Then $\Gamma$ dissects $P$ into a polygon $A$ and a congruent image of $A$. Hence $A$ is a reptile, because $A$ is the union of $2 k^{2}$ of the pieces $P_{i}$, which are similar to $P$. In the context of polyominoes this idea can be found for example in [3, p. 97] and [6, p. 52].

The last example in Fig. 1 is the so-called sphinx, which is a polyiamond composed by six equilateral triangles. It is a reptile with an odd number of vertices, whereas the number of vertices in all previous examples is even.
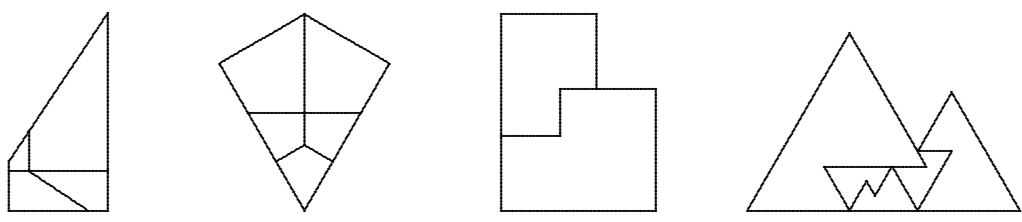

Fig. 2 Examples of irreptiles (see [9])

If a polygon $A$ splits into finitely many similar copies of $A$ that are not necessarily pairwise congruent then $A$ is called an irreptile (see [9]). Every irreptile $A$ gives rise to a dissection of the plane into images of $A$ under similarities whose similarity ratios are at least 1. Scherer's nice book [9] gives an insight into the great richness of irreptiles. Fig. 2 shows four examples. Further results on irreptiles appear sporadically in the literature or on the internet, mainly in the context of recreational mathematics and often concerning polyominoes or polyiamonds (see e.g. [8]).

In the present paper we describe several rather large classes of irreptiles, that are not polyominoes and, mostly, neither polyiamonds. These classes contain many examples from [9]. Our emphasis is on a large variety of shapes, but not on optimal dissections (i.e. into a minimal number of pieces). In some cases it will turn out that it is possible to obtain dissections into similar copies being based on proper similarities only. 
Irreptiles with many vertices cannot be convex. They have to have so-called reflex vertices where the size of the corresponding inner angle exceeds $\pi$. Indeed, if an irreptile has a total number of $v$ vertices then the number $v_{r}$ of reflex vertices is bounded by

$$
\frac{v}{2}-3<v_{r}<\frac{v}{2}-1
$$

(see [7, pp. 48-49], [4]). So if $v$ is even then $v_{r}=\frac{v}{2}-2$ and if $v$ is odd then $v_{r} \in$ $\left\{\frac{v-5}{2}, \frac{v-3}{2}\right\}$. As far as we know, in all known examples with odd $v$ one has $v_{r}=\frac{v-3}{2}$. Is $v_{r}=\frac{v-5}{2}$ possible? Does there exist a convex pentagon that is an irreptile?

In the sequel we use the symbols $\operatorname{cl}(A), \operatorname{int}(A), \operatorname{and} \operatorname{conv}(A)$ for denoting the closure, the interior, and the convex hull of a set $A \subseteq \mathbb{R}^{2}$, respectively.

\section{An uncountable family based on isosceles triangles}

Given a real parameter $\xi>0$, the origin $0=(0,0)$ together with the vectors $b_{1}=(1,0)$ and $b_{2}=\left(\frac{1}{2}, \xi\right)$ span an isosceles triangle. We fix moreover two integer parameters $0<k \leq l$. Then $c=k b_{1}+l b_{2}$ is the centre of the parallelogram $P=$ $\operatorname{conv}\left\{0,2 k b_{1}, 2 l b_{2}, 2 k b_{1}+2 l b_{2}\right\}$. We denote the reflection with respect to the point $c$ by $\delta_{c}$. Now we pick a simple polygonal arc $\Gamma$ with the following properties:

(i) $\Gamma$ connects the vertices $2 k b_{1}$ and $2 l b_{2}$ of $P$ and $\Gamma \backslash\left\{2 k b_{1}, 2 l b_{2}\right\} \subseteq \operatorname{int}(P)$,

(ii) all vertices of $\Gamma$ belong to the lattice $\mathbb{Z} b_{1}+\mathbb{Z} b_{2}=\left\{i b_{1}+j b_{2}: i, j \in \mathbb{Z}\right\}$ and all edges of $\Gamma$ are parallel to $b_{1}$ or $b_{2}-b_{1}=\left(-\frac{1}{2}, \xi\right)$,

(iii) $\Gamma$ is symmetric with respect to $c$, that is, $\Gamma=\delta_{c}(\Gamma)$, and

(iv) $\Gamma$ is contained in the triangle $T=\operatorname{conv}\left\{0,2 l b_{1}, 2 l b_{2}\right\}$.

Then $\Gamma$ splits $P$ into two polygons $A$ and $\delta_{c}(A)$, where $0 \in A$ without loss of generality (see Fig. 3).

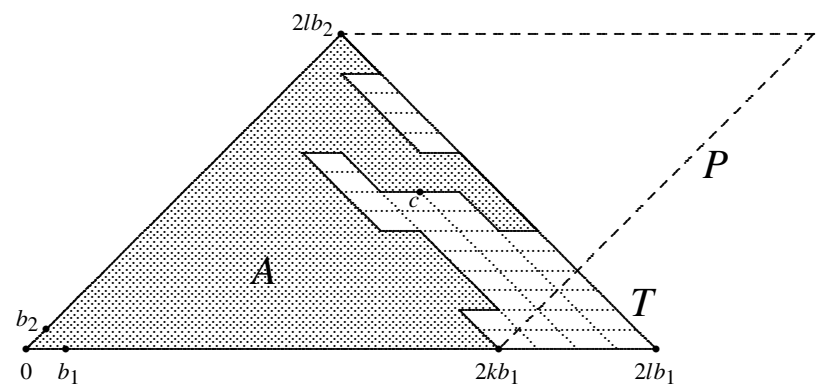

Fig. 3 Proof of Proposition 1

\section{Proposition 1.}

(a) The polygon A defined above is an irreptile. Moreover, if the length $\left\|b_{2}\right\|=\sqrt{\frac{1}{4}+\xi^{2}}$ is rational then there exists a dissection of A into finitely many images of $A$ under suitable proper similarities. 
(b) The number $v_{A}$ of vertices of $A$ is odd. If $v_{\Gamma}$ denotes the number of inner vertices of $\Gamma$ then $v_{A}=v_{\Gamma}+3$ and the number of reflex vertices of $A$ is $\frac{v_{\Gamma}}{2}$.

Proof. (a) A splits into proper congruent images of the triangle conv $\left\{0, b_{1}, b_{2}\right\}$, which itself is a proper similar copy of $T$. Hence it suffices to show that $T$ can be dissected into finitely many similar images of $A$. Since $A \subseteq T$, it is enough to show that $\operatorname{cl}(T \backslash A)$ has a dissection of that kind.

Both $A$ and $T$ have only one edge parallel to $b_{2}$, namely $\operatorname{conv}\left\{0,2 l b_{2}\right\}$. Hence the boundary of $\operatorname{cl}(T \backslash A)$ consists of line segments parallel to $b_{1}$ and $b_{2}-b_{1}$ only (see Fig. 3). Since all vertices of $\operatorname{cl}(T \backslash A)$ belong to the lattice $\mathbb{Z} b_{1}+\mathbb{Z} b_{2}=\mathbb{Z} b_{1}+\mathbb{Z}\left(b_{2}-b_{1}\right), \operatorname{cl}(T \backslash A)$ splits into finitely many translates of the parallelogram $P^{-}=\operatorname{conv}\left\{0, b_{1}, b_{2}-b_{1}, b_{2}\right\}$ (dotted in Fig. 3). Thus it remains to find a dissection of $P^{-}$into similar copies of $A$, which have to be proper if $\left\|b_{2}\right\|$ is rational.

The parallelogram $P^{+}=\operatorname{conv}\left\{0, b_{1}, b_{2}, b_{1}+b_{2}\right\}$ splits into $l k$ translates of

$$
\operatorname{conv}\left\{0, \frac{b_{1}}{l}, \frac{b_{2}}{k}, \frac{b_{1}}{l}+\frac{b_{2}}{k}\right\}=\frac{1}{2 k l} P=\frac{1}{2 k l}\left(A \cup \delta_{c}(A)\right) .
$$

$P^{-}$is the image of $P^{+}$under a reflection with respect to a vertical axis. This gives rise to a dissection of $P^{-}$into $2 \mathrm{kl}$ similar copies of $A$. So $A$ is an irreptile.

If $\left\|b_{2}\right\|=\frac{m}{n}, m, n \in\{1,2, \ldots\}$, is rational then there is a rotation $\gamma$ fixing 0 such that $\gamma\left(b_{2}\right)=-\left\|b_{2}\right\| b_{1}=-\frac{m}{n} b_{1}$ and hence $\gamma\left(b_{1}\right)=\frac{b_{2}-b_{1}}{\left\|b_{2}-b_{1}\right\|}=\frac{n}{m}\left(b_{2}-b_{1}\right)$. Now $P^{-}$is splitted into $\mathrm{kn}^{2} \mathrm{~lm} \mathrm{~m}^{2}$ translates of

$\operatorname{conv}\left\{0,-\frac{b_{1}}{k n^{2}}, \frac{b_{2}-b_{1}}{l m^{2}},-\frac{b_{1}}{k n^{2}}+\frac{b_{2}-b_{1}}{l m^{2}}\right\}=\gamma\left(\frac{1}{2 k l m n} P\right)=\gamma\left(\frac{1}{2 k l m n}\left(A \cup \delta_{c}(A)\right)\right)$.

This gives a dissection into proper similar images of $A$.

(b) $v_{A}=v_{\Gamma}+3$, because $0,2 k b_{1}$, and $2 l b_{2}$ are the only vertices of $A$ that are not inner vertices of $\Gamma$.

By (iii), $c$ is not a vertex of $\Gamma$ and the vertices of $\Gamma$ appear in pairs $\left(x, \delta_{c}(x)\right)$. So $v_{\Gamma}$ is even and $v_{A}$ is odd.

If $x$ is an inner vertex of $\Gamma$ then $x$ is a reflex vertex of $A$ if and only if $\delta_{c}(x)$ is a convex vertex of $A$. Thus the number of reflex vertices of $A$ is $\frac{v_{\Gamma}}{2}$.

Proposition 1 gives irreptiles with arbitrary odd numbers of vertices $v_{A} \geq 3$. In the following we modify the construction for obtaining even numbers $v_{A} \geq 4$.

We choose $\xi, b_{1}$, and $b_{2}$ as above and fix arbitrary integer parameters $k, l, m \geq 1$. Now we consider the parallelogram $P=\operatorname{conv}\left\{0,2(k+l) b_{1}, 2 m b_{2}, 2(k+l) b_{1}+2 m b_{2}\right\}$. $\delta_{c}$ denotes the reflection with respect to the centre $c=(k+l) b_{1}+m b_{2}$ of $P$. We pick a simple polygonal arc $\Gamma$ such that

(i) $\Gamma$ connects $(2 k+l) b_{1}$ with $l b_{1}+2 m b_{2}$ and $\Gamma \backslash\left\{(2 k+l) b_{1}, l b_{1}+2 m b_{2}\right\} \subseteq \operatorname{int}(P)$, 
(ii) all vertices of $\Gamma$ belong to the lattice $\mathbb{Z} b_{1}+\mathbb{Z} b_{2}$ and all edges of $\Gamma$ are parallel to $b_{1}$ or $b_{2}-b_{1}$, and

(iii) $\Gamma$ is symmetric with respect to $c$.

Again $\Gamma$ splits $P$ into two polygons $A$ and $\delta_{c}(A)$, where $0 \in A$ (see Fig. 4).
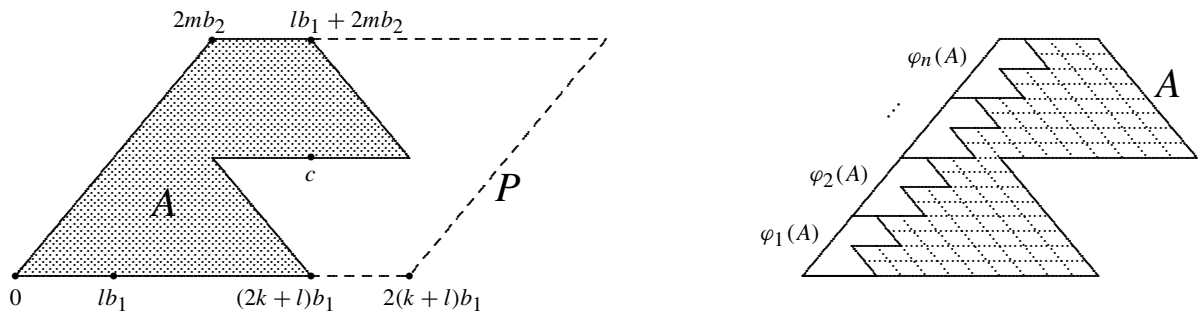

Fig. 4 Proof of Proposition 2

\section{Proposition 2.}

(a) The polygon A defined above is an irreptile. Moreover, if the length $\left\|b_{2}\right\|=\sqrt{\frac{1}{4}+\xi^{2}}$ is rational then there exists a dissection of A into finitely many images of $A$ under suitable proper similarities.

(b) The number $v_{A}$ of vertices of $A$ is even. If $v_{\Gamma}$ denotes the number of inner vertices of $\Gamma$ then $v_{A}=v_{\Gamma}+4$ and the number of reflex vertices of $A$ is $\frac{v_{\Gamma}}{2}$.

Proof. For every integer $n \geq 2$, the homothetic copies $\varphi_{i}(A)=\frac{1}{n} A+\frac{2 m(i-1)}{n} b_{2}, 1 \leq$ $i \leq n$, have pairwise disjoint interiors and cover the edge conv\{0,2mb $\}$ of $A$ (see the right-hand part of Fig. 4). We assume $n$ to be chosen large enough such that all $\varphi_{i}(A)$ are contained in $A$. Then the remaining polygon $\operatorname{cl}\left(A \backslash\left(\varphi_{1}(A) \cup \ldots \cup \varphi_{n}(A)\right)\right)$ is formed by vertices from the lattice $\frac{1}{n}\left(\mathbb{Z} b_{1}+\mathbb{Z} b_{2}\right)=\frac{1}{n}\left(\mathbb{Z} b_{1}+\mathbb{Z}\left(b_{2}-b_{1}\right)\right)$ and by edges parallel to $b_{1}$ and $b_{2}-b_{1}$ only. Thus we can decompose it into translates of $\frac{1}{n} P^{-}=\frac{1}{n} \operatorname{conv}\left\{0, b_{1}, b_{2}-\right.$ $\left.b_{1}, b_{2}\right\}$ (dotted in Fig. 4) and it suffices to prove that $P^{-}$admits a dissection into suitable similar copies of $A$. This remainder of part (a) and the verification of part (b) can be treated as in the proof of Proposition 1.

\section{A countable family of polyiamonds}

Let $b_{1}=(0,1)$ and $b_{2}=\left(\frac{1}{2}, \frac{\sqrt{3}}{2}\right)$ and fix arbitrary integers $k, l>0 . \delta_{c}$ is to denote the reflection with respect to the centre $c=k b_{1}+l b_{2}$ of the parallelogram $P=\operatorname{conv}\left\{0,2 k b_{1}, 2 l b_{2}, 2 k b_{1}+2 l b_{2}\right\}$. Let $\Gamma$ be a simple polygonal arc such that

(i) $\Gamma$ connects the vertices $2 k b_{1}$ and $2 l b_{2}$ of $P$ and $\Gamma \backslash\left\{2 k b_{1}, 2 l b_{2}\right\} \subseteq \operatorname{int}(P)$,

(ii) all vertices of $\Gamma$ belong to $\mathbb{Z} b_{1}+\mathbb{Z} b_{2}$ and all edges of $\Gamma$ are parallel to $b_{1}$ or $b_{2}-b_{1}=$ $\left(-\frac{1}{2}, \frac{\sqrt{3}}{2}\right)$, and

(iii) $\Gamma$ is symmetric with respect to $c$. 
$\Gamma$ dissects $P$ into two polygons $A$ and $\delta_{c}(A)$, where $0 \in A$ (see the left-hand part of Fig. 5). Note that this kind of polygon is closely related with that from the first part of the previous section. Here the parameter $\xi$ is restricted to $\frac{\sqrt{3}}{2}$, so that $A$ is a polyiamond. In contrast to that, the choice of $k$ and $l$ is more flexible, since $k>l$ is no longer forbidden, and the restriction (iv) from Section 2 is dropped.
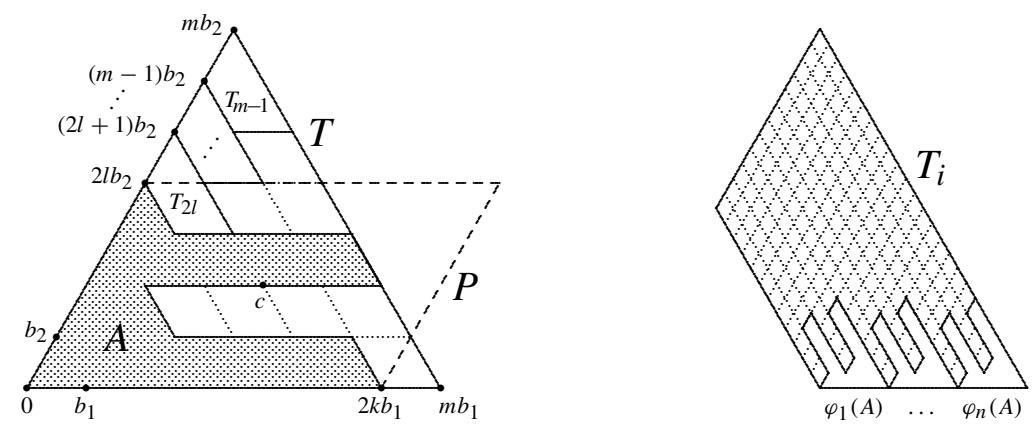

Fig. 5 Proof of Proposition 3

\section{Proposition 3.}

(a) The polygon A defined above is an irreptile. There exists a dissection of A into finitely many images of A under suitable proper similarities.

(b) The number $v_{A}$ of vertices of $A$ is odd. If $v_{\Gamma}$ denotes the number of inner vertices of $\Gamma$ then $v_{A}=v_{\Gamma}+3$ and the number of reflex vertices of $A$ is $\frac{v_{\Gamma}}{2}$.

Proof. Let $m$ be the smallest integer such that the equilateral triangle $T=\operatorname{conv}\left\{0, m b_{1}\right.$, $m b_{2}$ \} covers $A$. Since $A$ splits into finitely many equilateral triangles, it suffices to show that $T$ admits a dissection into finitely many proper similar copies of $A$.

In contrast to the situation of Proposition 1 , now $\operatorname{cl}(T \backslash A)$ has an edge $\operatorname{conv}\left\{2 l b_{2}, m b_{2}\right\}$ parallel to $b_{2}$. (It vanishes if $m=2 l$.) For every $i \in\{2 l, \ldots, m-1\}$, we define a trapezoid $T_{i}=\operatorname{conv}\left\{i b_{2},(i-1) b_{2}+b_{1},(i-1) b_{2}+2 b_{1},(i+1) b_{2}\right\}$. Then $\operatorname{cl}\left(T \backslash\left(A \cup T_{2 l} \cup \ldots \cup T_{m-1}\right)\right)$ has all its vertices in $\mathbb{Z} b_{1}+\mathbb{Z} b_{2}=\mathbb{Z} b_{1}+\mathbb{Z}\left(b_{2}-b_{1}\right)$ and all its edges are parallel to $b_{1}$ or $b_{2}-b_{1}$. We split it into parallelograms (dotted in the left-hand part of Fig. 5) and dissect them into proper similar copies of $A$ as we did in the proof of Proposition 1. Now it remains to prove that every $T_{i}, 2 l \leq i \leq m-1$, has a dissection of the same kind.

Let $\gamma$ be a rotation about the origin with angle $\frac{2 \pi}{3}$. The lower edge of $\gamma(A)$ is the only one parallel to $b_{1}$ and has length $2 l$. Hence, for every integer $n \geq 1$, there exist translates $\varphi_{j}(A), 1 \leq j \leq n$, of $\frac{1}{2 \ln } \gamma(A)$ such that the lower edge of $T_{i}$ splits into the lower edges of $\varphi_{1}(A), \ldots, \varphi_{n}(A)$. We assume $n$ to be fixed large enough such that all these translates are subsets of $T_{i}$ (see the right-hand part of Fig. 5). The remaining polygon $\operatorname{cl}\left(T_{i} \backslash\left(\varphi_{1}(A) \cup\right.\right.$ $\left.\left.\ldots \cup \varphi_{n}(A)\right)\right)$ is formed by vertices from $\frac{1}{2 l n}\left(\mathbb{Z} b_{2}+\mathbb{Z}\left(b_{2}-b_{1}\right)\right)$ and edges parallel to $b_{2}$ and $b_{2}-b_{1}$. Hence it splits into finitely many rhombs similar to conv $\left\{0, b_{2}, b_{2}-b_{1}, 2 b_{2}-b_{1}\right\}$ (dotted in Fig. 5). Dissections of these rhombs into proper similar copies of $A$ are obtained as in the proof of Proposition 1. This completes the verification of (a).

Claim (b) can be proved as in Proposition 1. 
One example of an irreptile found by the last construction is the sphinx (see the last example from Fig. 1). Proposition 3 says that a dissection representing the sphinx as an irreptile can be realized by the aid of proper similarities only (see Fig. 6 as an example). It is worth noting that this is impossible for the sphinx as a reptile.

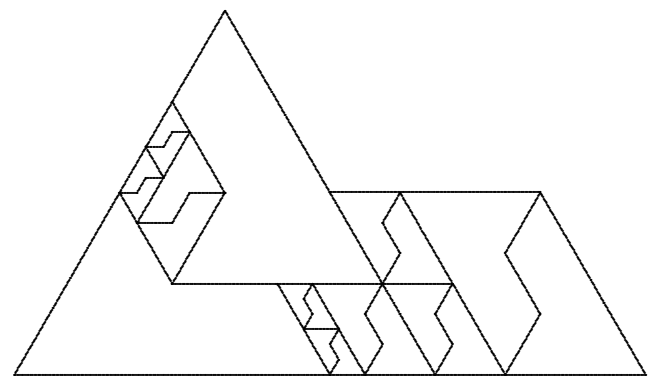

Fig. 6 A dissection of the sphinx based on proper similarities only

Proposition 4. Let the sphinx $S$ be dissected into $n \geq 2$ pairwise congruent similar copies $\varphi_{1}(S), \ldots, \varphi_{n}(S)$ of itself. Then at least one of the similarities $\varphi_{i}$ is an improper map.

Proof. Suppose that all $\varphi_{i}, 1 \leq i \leq n$, are proper. Let the vertices of $S$ be denoted by $a, \ldots, e$ as in Fig. 7. Then $b$ must be a vertex of one of the $\varphi_{i}(S)$, say of $\varphi_{1}(S)$. The inclusion $\varphi_{1}(S) \subseteq S$ is possible only if $b=\varphi_{1}(a)$ or $b=\varphi_{1}(b)$.

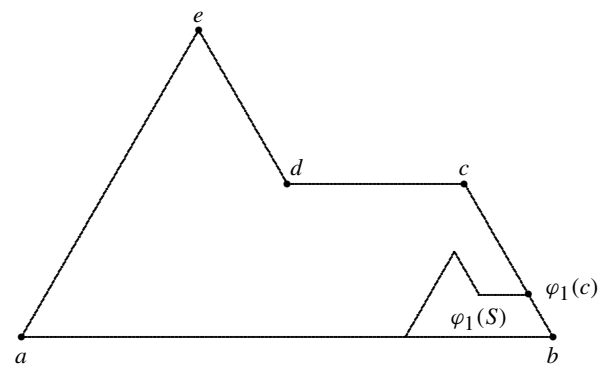

Case 1: $b=\varphi_{1}(b)$.

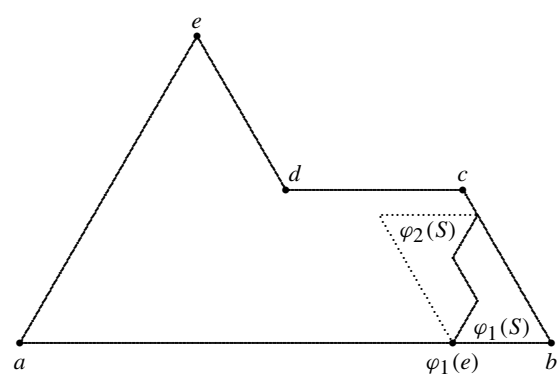

Case 2: $b=\varphi_{1}(a)$.

Fig. 7 Proof of Proposition 4

In the latter case (Case 1 in Fig. 7) $\varphi_{1}(c)$ had to be a vertex of one of the tiles $\varphi_{i}(S)$, $2 \leq i \leq n$. This is impossible, because all $\varphi_{i}(S)$ are proper congruent images of $\varphi_{1}(S)$.

In the remaining case $b=\varphi_{1}(a)$ (Case 2 in Fig. 7) the edge conv $\left\{\varphi_{1}(e), \varphi_{1}(d)\right\}$ of $\varphi_{1}(S)$ had to be an edge of another tile, say of $\varphi_{2}(S)$. Again using that $\varphi_{2}(S)$ is a proper congruent image of $\varphi_{1}(S)$ we conclude that the position of $\varphi_{2}(S)$ relative to $\varphi_{1}(S)$ is as it is illustrated by the dotted lines in Fig. 7 . 
Now $\varphi_{1}(e)$ plays a similar role in the remaining polygon $\varphi_{3}(S) \cup \ldots \cup \varphi_{n}(S)$ as the vertex $b$ did with respect to $S$. Repeated application of the above arguments shows that the horizontal strip of $S$ over the edge $\operatorname{conv}\{a, b\}$ whose height agrees with that of the parallelogram $P=\varphi_{1}(S) \cup \varphi_{2}(S)$ had to be dissected into translates of $P$, a contradiction.

Proposition 3 covers in particular a class of generalized sphinxes found by Hinrichs [5]. His family of examples served as a motivation for the present research.

We close this section with the remark that a suitable modification of the above construction yields polyiamond irreptiles with an even number of vertices. This coincides with the particular case of Proposition 2 where $\xi=\frac{\sqrt{3}}{2}$.

\section{A countable family based on isosceles right triangles}

We fix two integers $1 \leq l \leq k$ and consider the rectangle $R=\operatorname{conv}\left\{0,2 k e_{1}, 2 l e_{2}, 2 k e_{1}+\right.$ $\left.2 l e_{2}\right\}$, where $e_{1}=(1,0), e_{2}=(0,1) . \delta_{c}$ is to denote the reflection with respect to the centre $c=k e_{1}+l e_{2}$ of $R$. Let $\Gamma$ be a simple polygonal arc such that

(i) $\Gamma$ connects $2 k e_{1}$ with $2 l e_{2}$ and $\Gamma \backslash\left\{2 k e_{1}, 2 l e_{2}\right\} \subseteq \operatorname{int}(R)$,

(ii) all vertices of $\Gamma$ belong to $\mathbb{Z} \times \mathbb{Z}$ and all edges of $\Gamma$ are parallel to $e_{1}+e_{2}$ or $e_{1}-e_{2}$,

(iii) $\Gamma$ is symmetric with respect to $c$, and

(iv) $\Gamma$ is contained in the triangle $T=\operatorname{conv}\left\{0,2 k e_{1}, 2 k e_{2}\right\}$.

$\Gamma$ dissects $R$ into two polygons $A$ and $\delta_{c}(A)$, where $0 \in A$ (see the left-hand part of Fig. 8). In contrast to the previous examples, the present family contains polygons $A$ whose edges have four different directions.
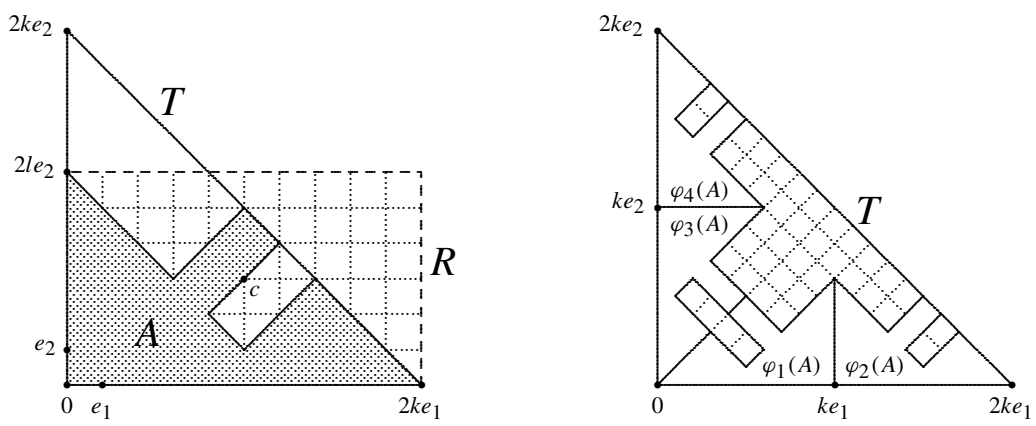

Fig. 8 Proof of Proposition 5

\section{Proposition 5.}

(a) The polygon A defined above is an irreptile.

(b) The number $v_{A}$ of vertices of $A$ is odd. If $v_{\Gamma}$ denotes the number of inner vertices of $\Gamma$ then $v_{A}=v_{\Gamma}+3$ and the number of reflex vertices of $A$ is $\frac{v_{\Gamma}}{2}$. 
Proof. It suffices to show that $T$ admits a dissection into finitely many similar copies of $A$, because $A$ splits into isosceles right triangles. We choose similarities $\varphi_{1}, \ldots, \varphi_{4}$ with similarity ratio $\frac{1}{2}$ such that $T=\varphi_{1}(T) \cup \ldots \cup \varphi_{4}(T)$ and $\varphi_{1}(0)=\varphi_{2}(0)=k e_{1}$, $\varphi_{3}(0)=\varphi_{4}(0)=k e_{2}, \varphi_{1}\left(2 k e_{1}\right)=\varphi_{3}\left(2 k e_{1}\right)=0, \varphi_{2}\left(2 k e_{1}\right)=2 k e_{1}, \varphi_{4}\left(2 k e_{1}\right)=2 k e_{2}$. Then the remainder $\operatorname{cl}\left(T \backslash\left(\varphi_{1}(A) \cup \ldots \cup \varphi_{4}(A)\right)\right)$ has all its vertices in $\frac{1}{2}(\mathbb{Z} \times \mathbb{Z})$ and all its edges are parallel to $e_{1}+e_{2}$ or $e_{1}-e_{2}$ (see the right-hand part of Fig. 8). Hence this remainder splits into squares (dotted in the illustration) which can be dissected into similar copies of $R=A \cup \delta_{c}(A)$. This proves (a).

Claim (b) can be verified as in Proposition 1.

\section{An uncountable family related to rhombs}

Let $b_{1}$ and $b_{2}$ be two vectors spanning a rhomb, that is, $b_{1}$ and $b_{2}$ are linearly independent and of the same length. Given an integer $k \geq 0$, we define $A$ as the polygon bounded by the simply closed polygonal arc connecting $0,(2 k+1) b_{1},(2 k+1) b_{1}+b_{2}, 2 k b_{1}+b_{2}$, $2 k b_{1}+2 b_{2}, \ldots,(k+1) b_{1}+k b_{2},(k+1) b_{1}+(k+1) b_{2}, 0$ (see the left-hand part of Fig. 9).
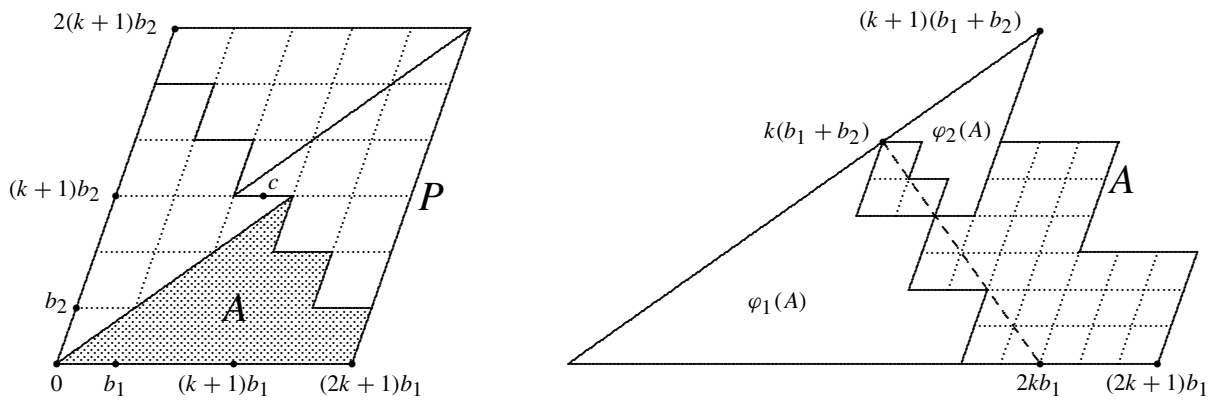

Fig. 9 Proof of Proposition 6

Proposition 6. The polygon A defined above is an irreptile. Among the $2 k+3$ vertices of $A$ there are $k$ reflex vertices.

Proof. Let $\gamma$ be the reflection with respect to the axis $\mathbb{R}\left(b_{1}+b_{2}\right)=\left\{\xi\left(b_{1}+b_{2}\right): \xi \in \mathbb{R}\right\}$ and let $\delta_{c}$ be the reflection with respect to the centre $c=\left(k+\frac{1}{2}\right) b_{1}+(k+1) b_{2}$ of the parallelogram $P=\operatorname{conv}\left\{0,(2 k+1) b_{1}, 2(k+1) b_{2},(2 k+1) b_{1}+2(k+1) b_{2}\right\}$. Then $P$ is dissected into $A, \gamma(A), \delta_{c}(A)$, and $\delta_{c} \gamma(A)$ (see the left-hand part of Fig. 9).

The images $\varphi_{1}(A)=\frac{k}{k+1} A$ and $\varphi_{2}(A)=-\frac{1}{k+1} \gamma(A)+(k+1)\left(b_{1}+b_{2}\right)$ are contained in $A$, cover the edge conv $\left\{0,(k+1)\left(b_{1}+b_{2}\right)\right\}$ of $A$, and have disjoint interiors, because they are separated by the line through $2 k b_{1}$ and $k\left(b_{1}+b_{2}\right)$ (see the right-hand part of Fig. 9). Hence $A$ splits into $\varphi_{1}(A), \varphi_{2}(A)$, and finitely many rhombs similar to conv $\left\{0, b_{1}, b_{2}, b_{1}+b_{2}\right\}$. Any of these rhombs can be dissected into finitely many similar copies of $P=A \cup \gamma(A) \cup$ $\delta_{c}(A) \cup \delta_{c} \gamma(A)$. So $A$ is an irreptile.

The vertices of $A$ can easily be counted. In particular, $(2 k+1-i) b_{1}+i b_{2}, 1 \leq i \leq k$, are reflex vertices. 
Proposition 6 gives irreptiles with an odd number of vertices. Even numbers can be obtained as follows. We fix $b_{1}, b_{2}$, and $k$ as above and define $A$ as the polygon bounded by the polygonal arc $0,(2 k+2) b_{1},(2 k+2) b_{1}+b_{2},(2 k+1) b_{1}+b_{2},(2 k+1) b_{1}+2 b_{2}, \ldots$, $(k+2) b_{1}+k b_{2},(k+2) b_{1}+(k+1) b_{2},(k+1) b_{1}+(k+1) b_{2}, 0$ (see the left-hand part of Fig. 10).
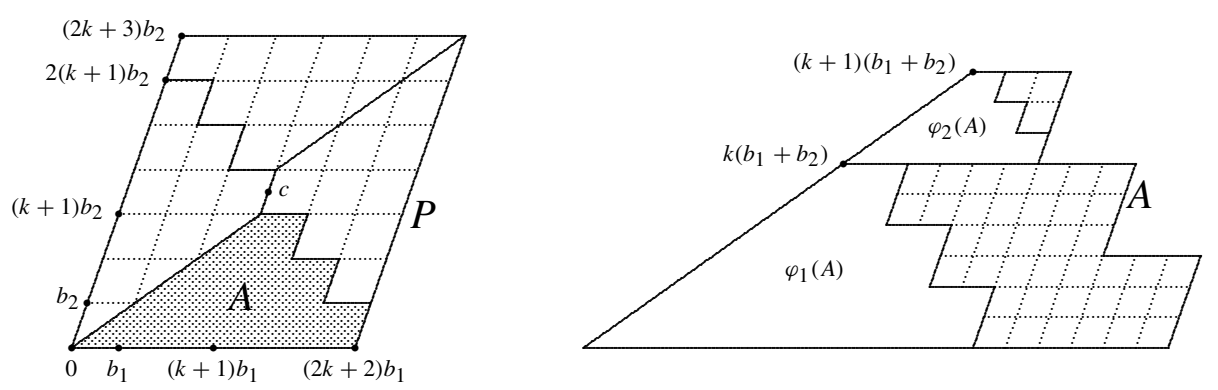

Fig. 10 Proof of Proposition 7

Proposition 7. The polygon A defined above is an irreptile. Among the $2 k+4$ vertices of $A$ there are $k$ reflex vertices.

Proof. We refer to Fig. 10 and leave the details to the reader.

\section{A countable family of non-lattice pentagons}

Each of the previously defined irreptiles has its vertices in some lattice $\mathbb{Z} b_{1}+\mathbb{Z} b_{2}$. In the following we describe a family of pentagons including infinitely many non-lattice members. We use the following technical tool.

Lemma. Let $P_{\lambda}$ and $P_{\mu}$ be two parallelograms with pairwise parallel sides, $P_{\lambda}$ having edges of lengths 1 and $\lambda$ and $P_{\mu}$ having edges of lengths 1 and $\mu$. If there are an integer $m \geq 0$ and rational numbers $r_{0}, \ldots, r_{m}$ with $r_{0} \geq 0$ and $r_{i}>0$ for $1 \leq i \leq m$ such that

$$
\lambda=r_{0} \mu+\frac{1}{r_{1} \mu+\frac{1}{\ddots \cdot+\frac{1}{r_{m} \mu}}}
$$

then $P_{\lambda}$ can be dissected into finitely many similar copies of $P_{\mu}$.

Proof. Theorem 5 from [1] includes the above claim for rectangles. The generalization to parallelograms is obvious.

Proposition 8. Let $\xi, \eta$ be real numbers with $0<\eta<\xi$ and $\xi+\eta<2$ such that there are integers $m, n \geq 0$ and rational numbers $p_{0}, \ldots, p_{m}, q_{0}, \ldots, q_{n}$ with $p_{0}, q_{0} \geq 0$ and 


$$
\begin{aligned}
& p_{1}, \ldots, p_{m}, q_{1}, \ldots, q_{n}>0 \text { satisfying } \\
& 2 \eta=p_{0} \xi+\frac{1}{p_{1} \xi+\frac{1}{\ddots \cdot+\frac{1}{p_{m} \xi}}} \text { and } \quad \frac{2 \eta(\xi+\eta)}{\xi-\eta}=q_{0} \xi+\frac{1}{q_{1} \xi+\frac{1}{\ddots \cdot+\frac{1}{q_{n} \xi}}} \text {. }
\end{aligned}
$$

Moreover, let the pentagon A be obtained by cutting off a parallelogram of edge lengths $\frac{1}{2}$ and $\eta$ from an isosceles triangle $T$ with edges of lengths 1,1 , and $\xi+\eta$ as illustrated in Fig. 11. Then $A$ is an irreptile.
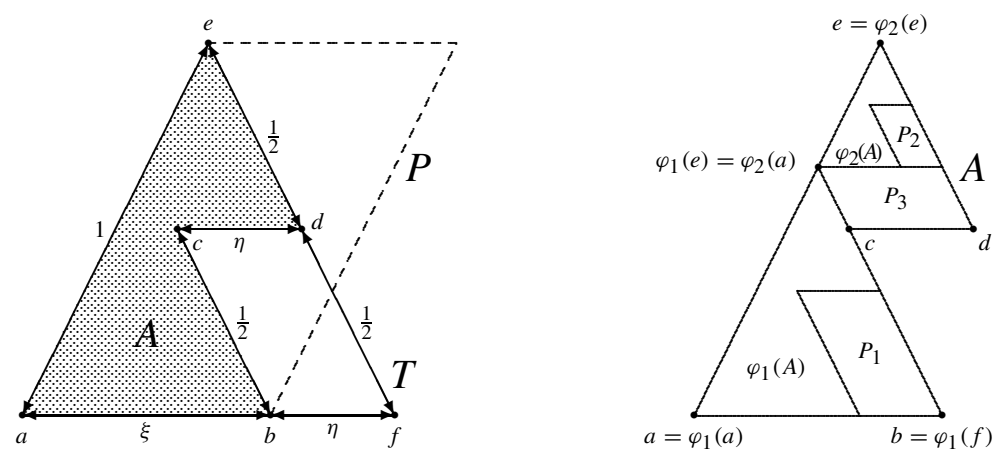

Fig. 11 Proof of Proposition 8

Proof. We use $a, b, c, d, e, f$ for denoting the vertices of $A$ and $T$ as in Fig. 11. $\delta$ is to denote the reflection with respect to the centre of the segment from $c$ to $d$. Then $A$ and $\delta(A)$ form a dissection of a parallelogram $P$ with edges of lengths 1 and $\xi$.

Let $\varphi_{1}$ and $\varphi_{2}$ be dilatations with fixed points $a$ and $e$, respectively, such that $\varphi_{1}(f)=b$ and $\varphi_{2}(a)=\varphi_{1}(e)$. The similarity ratio of $\varphi_{1}$ is $\frac{\|b-a\|}{\|f-a\|}=\frac{\xi}{\xi+\eta}$. Now $A$ splits into $\varphi_{1}(A)$, $\varphi_{2}(A)$, and three parallelograms $P_{1}, P_{2}, P_{3}$ with angles of the same sizes as those of $P$ (see the right-hand part of Fig. 11).

Both $P_{1}$ and $P_{2}$ are similar to the parallelogram conv $\{b, c, d, f\}$. Hence in both cases the ratio of the edge lengths is $1: 2 \eta$. By the lemma, the first technical assumption above guarantees that $P_{1}$ and $P_{2}$ can be dissected into finitely many similar copies of $P=A \cup \delta(A)$.

The lengths of the edges of $P_{3}$ are $\|d-c\|=\eta$ and

$$
\begin{aligned}
\left\|\varphi_{1}(e)-c\right\| & =\left\|\varphi_{1}(e)-\varphi_{1}(f)\right\|-\|c-b\| \\
& =\frac{\xi}{\xi+\eta}\|e-f\|-\|c-b\|=\frac{\xi}{\xi+\eta}-\frac{1}{2}=\frac{\xi-\eta}{2(\xi+\eta)} .
\end{aligned}
$$

Hence $P_{3}$ is similar to a parallelogram with edges of lengths 1 and $\frac{\|d-c\|}{\left\|\varphi_{1}(e)-c\right\|}=\frac{2 \eta(\xi+\eta)}{\xi-\eta}$. The second technical assumption and the lemma show that $P_{3}$ admits a finite dissection into similar copies of $P=A \cup \delta(A)$, too. This completes the proof. 

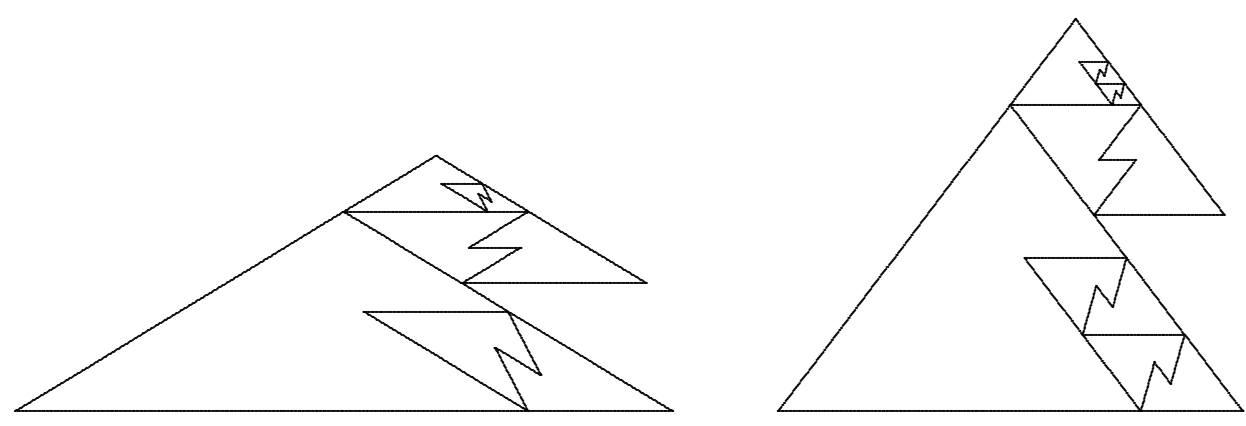

Fig. 12 Two non-lattice examples

For obtaining a class of particular examples, we consider the parameters $m=1, p_{0}=0$, $p_{1}=k \in\{1,2, \ldots\}, n=0$, and $q_{0}=1$. This gives $\xi=\frac{2}{\sqrt{2 k(\sqrt{17}-3)}}$ and $\eta=\frac{\sqrt{17}-3}{4} \xi$, in particular $0<\eta<\xi$ and $\xi+\eta<2$ for all $k \in\{1,2, \ldots\}$. All these examples are non-lattice polygons, because $\frac{\|d-c\|}{\|b-a\|}=\frac{\eta}{\xi}=\frac{\sqrt{17}-3}{4}$ is irrational. Fig. 12 shows the cases $k=1$ and $k=2$.

\section{An uncountable family of trapezoids}

In [9, Chapter 4] Scherer introduces trapezoids $H(\xi, \eta)$ whose parallel edges of lengths $\xi$ and $\eta$ are perpendicular to a third edge of length 1 (see Fig. 13). He shows that, for every $\xi>0, H\left(\xi, \frac{1}{\xi}\right)$ splits into four smaller similar copies of $H\left(\xi, \frac{1}{\xi}\right)$. This gives another family of non-lattice irreptiles. The example with $\xi=2$ is illustrated in Fig. 2.

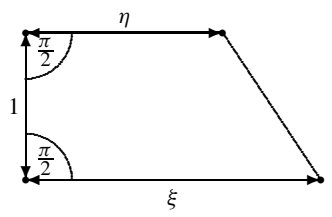

Fig. 13 The trapezoid $H(\xi, \eta)$

Moreover, Scherer shows that $H(\xi, \eta)$ is an irreptile if both $\xi$ and $\eta$ are rational. This admits the following generalization.

Proposition 9. If the ratio $\lambda$ of the lengths of the parallel edges of a trapezoid $T$ satisfies $\frac{\lambda}{(\lambda+1)^{2}}=\frac{m}{n}$ with $m, n \in\{1,2, \ldots\}$, then there exists a dissection of $T$ into $2(m+n+1)$ proper similar copies of $T$.

Proof. Suppose that the parallel edges are horizontal and have the lengths $\lambda$ and 1 without loss of generality. Fig. 14 illustrates the required dissection of $T . P$ is a parallelogram formed by two congruent copies of $T$. The length of the horizontal edges of $P$ is $\lambda+1$. 


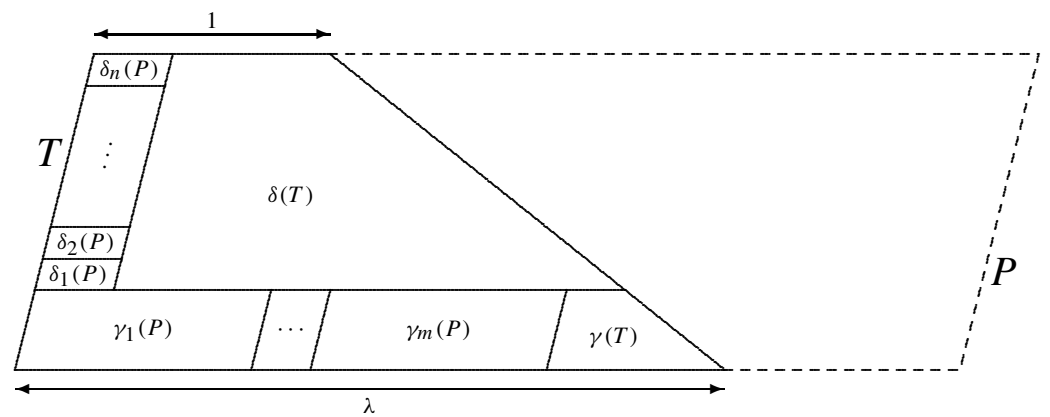

Fig. 14 Proof of Proposition 9

The similarity ratio of the maps $\gamma, \gamma_{1}, \ldots, \gamma_{m}$ is $\varrho_{1}=\frac{\lambda}{\lambda+m(\lambda+1)}$. Hence the length of the lower edge of the trapezoid $\gamma(T) \cup \gamma_{1}(P) \cup \ldots \cup \gamma_{m}(P)$ coincides with that of the lower edge of $T$. For $\delta$, we choose a ratio $\varrho_{2}=1-\varrho_{1}=\frac{m(\lambda+1)}{\lambda+m(\lambda+1)}$, so that the heights of $\gamma(T) \cup \gamma_{1}(P) \cup \ldots \cup \gamma_{m}(P)$ and of $\delta(T)$ add up to that of $T$. Finally, $\delta_{1}, \ldots, \delta_{n}$ use the ratio $\varrho_{3}=\frac{\varrho_{2}}{n}=\frac{m(\lambda+1)}{n(\lambda+m(\lambda+1))}$. Hence $\delta(T) \cup \delta_{1}(P) \cup \ldots \cup \delta_{n}(P)$ is a trapezoid, too. It remains to show that the length of its upper edge agrees with that of $T$, that is, $1 \varrho_{2}+(\lambda+1) \varrho_{3}=1$. One easily checks this by the aid of the assumption $\frac{\lambda}{(\lambda+1)^{2}}=\frac{m}{n}$.

\section{References}

[1] Freiling, C.; Laczkovich, M.; Rinne, D.: Rectangling a rectangle. Discrete Comput. Geom. 17 (1997), $217-225$.

[2] Golomb, S.W.: Replicating figures in the plane. Math. Gaz. 48 (1964), 403-412.

[3] Golomb, S.W.: Polyominoes: puzzles, patterns, problems, and packings. Princeton University Press, Second edition, 1994.

[4] Hertel, E.: Private communication. Jena 2007.

[5] Hinrichs, A.: Private communication. Heidelberg 2004.

[6] Martin, G.E.: Polyominoes: a guide to puzzles and problems in tiling. Mathematical Association of America, 1991.

[7] Osburg, I.: Selbstähnliche Polyeder. Ph.D. thesis, Friedrich-Schiller-Universität Jena, 2004.

[8] Reid, M.: Tiling with similar polyominoes. J. Recreational Math. 31 (2002-2003), 15-24.

[9] Scherer, K.: A Puzzling Journey To The Reptiles And Related Animals. Privately published (see http://karl.kiwi.gen.nz/bkrintro.html), Auckland, New Zealand, 1987.

Christian Richter

Institute of Mathematics

Friedrich-Schiller-University

D-07737 Jena, Germany

e-mail: christian.richter@uni-jena.de

This research was supported by DFG grant RI 1087/3. 